\title{
MICROBIAL FUEL CELL OPERATION WITH PERIODIC CONNECTION OF THE EXTERNAL RESISTANCE
}

\author{
F. Grondin ${ }^{\mathrm{a}, \mathrm{b}}$, M. Perrier ${ }^{\mathrm{b}}$, B. Tartakovsky ${ }^{\mathrm{a}, \mathrm{b}}$ \\ ${ }^{a}$ Biotechnology Research Institute, National Research Council, 6100 Royalmount Ave., \\ Montréal, Que., Canada H4P 2R2 (Boris.Tartakovsky@cnrc-nrc.gc.ca Tel: 1-514-496-2664) \\ ${ }^{b}$ Departement de Génie Chimique, École Polytechnique Montréal, \\ C.P.6079 Succ., Centre-Ville Montréal, Que., Canada H3C 3A7 (e-mails: felix.grondin@polymtl.ca; \\ michel.perrier@polymtl.ca)
}

\begin{abstract}
Laboratory-scale microbial fuel cells are often operated with a constant electrical load. Meanwhile, variations in influent strength and quality, as well as the processes of biofilm growth and decay lead to significant changes of the MFC internal resistance over time. This inevitably results in a mismatch between the internal and the external resistances therefore decreasing MFC power output. This study presents the concept of MFC operation with intermittent connection of the electrical load (external resistance). The efficiency of the proposed method is demonstrated by MFC operation with external resistances significantly below the MFC internal resistance and comparing power outputs obtained using either constant or intermittently connected resistances.
\end{abstract}

Keywords: Microbial fuel cell; Electrical load; Intermittent connection; Duty cycle.

\section{INTRODUCTION}

Recent research efforts dedicated to improving microbial fuel cell (MFC) design, materials, and the knowledge of the microbial community have increased MFC power output by several orders of magnitude (Logan and Regan 2006; Lovley 2006; Rismani-Yazdi et al. 2008; Rozendal et al. 2008). However, an aspect that has been overlooked is how the produced energy is harvested. MFC power output is maximized when the electrical load (external resistance) connected to the cell is equal to the total internal resistance (Aelterman et al. 2008; Logan 2008; Woodward et al. 2009). In laboratory tests, MFCs are often operated with a constant external resistance. Meanwhile, variations in operating conditions and the processes of biofilm growth and decay lead to significant changes of the internal resistance over time. This inevitably results in a mismatch between the internal and the external resistances therefore decreasing MFC power output.

In a laboratory, the resistance mismatch problem can be managed by manually adjusting the variable external resistor connected to the MFC. This method requires a knowledge of MFC internal resistance, which can be estimated in polarization tests (Aelterman et al. 2008). However, the manual external resistance adjustments are infrequent, e.g. weekly or daily, while the internal resistance could significantly change within minutes, thus leading to significant energy losses. Recently, Woodward et al (2010) proposed a method for external resistance control, which uses an on-line perturbation/observation $(\mathrm{P} / \mathrm{O})$ algorithm for maximizing the power output. Also, a logic-based control approach for adjusting the external resistance was recently proposed (Premier et al. 2011). Both methods provide real-time optimization of the external resistance, however the practical implementation of these methods would require a device with an electrical load that can be changed on demand. Meanwhile, the electrical loads are not always adjustable.

When controlling electrical motors and other electrical devices, the average applied power is often controlled by periodically turning on and off the switch between the supply and the load. This mode of operation is described by a duty cycle, defined as a portion of time during which the power source is connected to the device. The duty cycle can be used to adjust the electrical load and match the resistances (impedances). A similar approach is often used in maximum power point tracking systems for efficient harvesting of renewable energy (Esram and Chapman 2007; Koutroulis and Kalaitzakis 2006) where a converter is introduced between the power source and the device. In this case the duty cycle is used to adjust the load and match the impedances. Indeed, the converter can be seen as an equivalent resistance added to the non-controllable electrical load with the value of this equivalent resistance being a function of the duty cycle.

The approach of controlling the equivalent resistance by duty cycle adjustment could be also used in MFC operation, however it requires a thorough evaluation, since the performance of the electricigenic microorganisms under a varying electrical load is unknown. This study presents the concept of MFC operation with intermittent connection of the electric load (R-periodic operation) and describes the experimental results, which confirm the efficiency of the proposed method.

\section{MATERIALS AND METHODS}

\subsection{MFC design and operation}


A membrane-less air-cathode MFC shown in Fig. 1 was constructed using nylon plates. The anode was made of $5 \mathrm{~mm}$ thick carbon felt measuring $10 \mathrm{~cm} \times 5 \mathrm{~cm}$ (SGL Canada, Kitchener, ON, Canada) and the cathode was a gas diffusion electrode with a Pt load of $0.5 \mathrm{mg} \mathrm{cm}^{-2}$ (GDE LT 120EW, ETEK Division, PEMEAS Fuel Cell Technologies, Somerset, NJ, USA). The electrodes were separated by a nylon cloth with a thickness of about $0.5 \mathrm{~mm}$. An external recirculation loop was used for anodic liquid mixing. The anodic chamber temperature was maintained at $25^{\circ} \mathrm{C}$. The MFC was continuously fed with the acetate stock solution and a trace metals solution. A hydraulic retention time of $5 \mathrm{~h}$ was maintained.

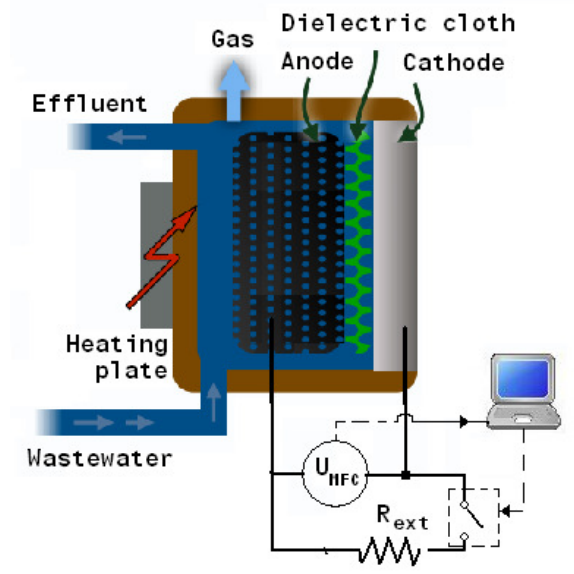

Figure 1. Experimental setup.

Intermittent connection of an external resistor $\left(R_{\text {ext }}\right)$ to $\mathrm{MFC}$ terminals was achieved by adding an electronic switch (IRF540, International Rectifier, El Sequndo, CA, USA) to the external electrical circuit. The switch was computer-controlled using a Labjack U12 data acquisition board (LabJack Corp., Lakewood, CO, USA). The data acquisition board was also used to record MFC voltage.

MFC operation with intermittent connection of an electrical load can be described by a duty cycle $(D)$ as:

$$
D=\frac{t_{\text {on }}}{t_{\text {off }}+t_{\text {on }}}
$$

where $t_{\text {on }}$ is the "on" time within each cycle during which the electrical load (external resistance) is connected and $t_{\text {off }}$ is the disconnection ("off") time. The cycle time $\left(T_{\text {cyc }}\right)$ can be expressed as $T_{\text {cyc }}=t_{\text {on }}+t_{\text {off }}$.

MFC operation with an adjustable duty cycle was achieved by switching between an "on" and an "off" states based on voltage measurements at MFC terminals. The following algorithm was used. $R_{\text {ext }}$ was disconnected, if $U_{\text {MFC }}$ decreased below a pre-defined minimum voltage threshold $\left(U_{\min }\right)$, then
$R_{\text {ext }}$ was re-connected once $U_{\mathrm{MFC}}$ exceeded a predefined maximum voltage threshold $\left(U_{\max }\right)$.

\subsection{Electrochemical measurements and performance} characterization

Polarization tests (PTs) were carried out to estimate the total internal resistance $\left(R_{\text {int }}\right)$ of the MFC. In each PT, $R_{\text {ext }}$ was disconnected for $30 \mathrm{~min}$, then open circuit voltage (OCV) was measured. Subsequently, the external resistance was reconnected and step-wise decreased every 10 minutes from $1000 \Omega$ to $5-15 \Omega$ to obtain 7-10 measurements. Voltage $(U)$ was measured at the end of each 10 min period. Stable voltage readings were assured by comparing voltage values during the last 3 minutes of each period. The resulting voltage and current values were used to construct polarization curves, i.e. voltage vs. current plots from where the MFC's total (ohmic and solution) $R_{i n t}$ was estimated by the slope of the linear region (Fan et al. 2008).

During MFC operation with constantly connected $R_{\text {ext }}$, power output $\left(P_{\text {out }}, \mathrm{mW} \mathrm{L}_{\mathrm{A}}^{-1}\right)$ was calculated using measurements of output voltage and a known value of $R_{\text {ext }}$. During MFC operation with intermittently connected $R_{\text {ext }}$ an average power output per cycle $\left(P_{a v}\right)$ was calculated as

$$
P_{a v}=1 / T_{c y c} \int_{0}^{T c y c}\left(U^{2}(t) / R_{\text {ext }}\right) d t
$$

where $U(t)$ is the voltage measured at $R_{\text {ext }}$ at time $t$.

The apparent total internal capacitance of the electrode surface was estimated by analyzing cyclic voltammograms obtained at different sweep rates (Trasatti and Petrii 1991). Cyclic voltammetry was performed using an electrochemical analyzer CHI 601A (CH Instruments, Austin, TX). A two-electrode setup was used (anode as a working electrode and a cathode as a counter and reference electrode). Also, the measurements were repeated with the cathode as a working electrode and the anode as a counter and reference electrode. The voltammograms were performed around the OCV value estimated as described above at scan rates between $1-15 \mathrm{mV}$ $\mathrm{s}^{-1}$. Current measurements at a voltage corresponding to MFC OCV were plotted against the scan rate and the apparent internal capacitance was estimated as a slope of the linear part of this curve.

\subsection{Factorial design experiment}

The full factorial design experiment aimed at optimizing $P_{\mathrm{av}}$ was performed by varying the periodic operation voltage threshold $\left(U_{\min }\right.$ and $\left.U_{\max }\right)$ between low, intermediate and high values. The plan necessitated 9 different trials. The experiment used a $10 \Omega$ external load, and contained 3 additional centre points to assess measurements variability.

\section{RESULTS AND DISCUSSION}

\subsection{Internal resistance and capacitance estimations}

Several polarization tests were conducted to estimate the MFC internal resistance, both during MFC operation at a "normal" organic loading rate (OLR) of $4 \mathrm{~g} \mathrm{~L}_{\mathrm{A}}^{-1} \mathrm{~d}^{-1}$ (corresponding to 
$1000 \mathrm{mg} \mathrm{L}^{-1}$ influent concentration) and at a "low" OLR of $1 \mathrm{~g}$ $\mathrm{L}_{\mathrm{A}}^{-1} \mathrm{~d}^{-1}$ (250 mg L $\mathrm{m}^{-1}$ influent concentration). The corresponding acetate concentrations in the anodic chamber were 170-200 $\mathrm{mg} \mathrm{L}^{-1}$ and $20-40 \mathrm{mg} \mathrm{L}^{-1}$, for normal and low OLRs, respectively.

At a normal OLR, $R_{\text {int }}$ estimations varied between $19-24 \Omega$ (Fig. 2A). These estimations are in agreement with an external resistance of $18-25 \Omega$ used during MFC operation by the computer-controlled perturbation/observation algorithm, which maximized MFC power output by real time external resistance correction (Pinto et al. 2011; Woodward et al. 2009).

MFC operation at a reduced organic load led to an increase of $R_{\text {int }}$ to $50-70 \Omega$, as can be seen from the analysis of the polarization curves shown Fig. 2A. Also, acetate limitation led to a power output decrease from $2.1 \pm 0.2 \mathrm{~mW}$ to $0.8 \pm 0.1$ $\mathrm{mW}$. Overall, the polarization tests demonstrated a strong dependence of $R_{\text {int }}$ on the carbon source concentration in the anodic chamber. A similar dependence was observed in a MFC fed with synthetic wastewater (Pinto et al. 2011).
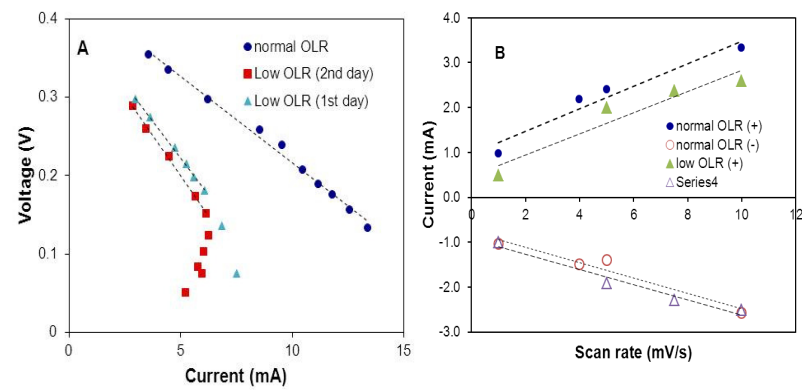

Figure 2. (A) polarization curves and (B) current vs scan rate diagrams obtained under normal OLR $\left(4 \mathrm{~g} \mathrm{~L}_{\mathrm{A}}^{-1} \mathrm{~d}^{-1}\right)$ and low OLR ( $\left.1 \mathrm{~g} \mathrm{~L}_{\mathrm{A}}^{-1} \mathrm{~d}^{-1}\right)$ conditions. $\mathrm{R}_{\text {int }}$ estimations were 22 , 41, and $38 \Omega$ for normal OLR, low OLR (day 1) and low OLR (day 2) tests respectively. Apparent capacitance estimations were 0.20 $\pm 0.08 \mathrm{~F}$ and $0.20 \pm 0.09 \mathrm{~F}$ for normal and low OLR, respectively.

Estimation of an MFC's apparent capacitance was carried out using the cyclic voltammetry (CV) technique. After cyclic voltammograms were obtained at different scan rates, the current in the middle of the potential range was plotted as a function of the sweep rate to estimate the slope, as shown in Figure 2B. The cyclic voltammograms were acquired at OLRs of 4 and $1 \mathrm{~g} \mathrm{~L} \mathrm{~L}_{\mathrm{R}}^{-1} \mathrm{~d}^{-1}$ and apparent capacitance estimations of $0.20 \pm 0.08 \mathrm{~F}$ and $0.20 \pm 0.09 \mathrm{~F}$, were obtained for normal and low OLRs, respectively. Although the apparent capacitance estimations obtained by cyclic voltammetry have a number of known limitations and can be biased (Trasatti and Petrii 1991), the estimations pointed out to a significant internal capacitance of the MFC. This capacitance might be attributed to the high surface area of the carbon felt anode (Chen et al. 2011).

\section{2 -periodic $M F C$ operation}

Recently, Donovan et al (2008) proposed an MFC power management system, which included a capacitor for energy accumulation and a DC-DC converter. This system was operated by accumulating the electrical energy in the capacitor for several dozens of minutes and then dispensing it in a burst of high power. This approach, however, did not address the problem of MFC operation at external resistance (impedance) values, which are significantly lower than the MFC internal resistance.

Here, we hypothesized that the considerable internal capacitance of the MFC can be used to resolve the resistance mismatch problem by MFC operation with periodic connection and disconnection of the electrical load (external resistance) at a relatively high frequency. The proposed method of MFC operation was tested with $R_{\text {ext }}=10 \Omega$, which was chosen to be below the estimated internal resistance of 19 - $24 \Omega$. The connection $\left(t_{\text {on }}\right)$ and disconnection $\left(t_{\text {off }}\right)$ times were set to $5 \mathrm{~s}$ and $2 \mathrm{~s}$, respectively. The test was carried out under acetate non-limiting conditions (i.e. OLR $=4 \mathrm{~g} \mathrm{~L}_{\mathrm{A}}^{-1} \mathrm{~d}^{-1}$ ). Fig. 3 shows MFC voltage $\left(U_{\mathrm{MFC}}\right)$ measured at the electrode terminals and the current at $R_{\text {ext }}$ calculated as $I_{\text {ext }}=U_{\text {ext }} / R_{\text {ext }}$, where $U_{\text {ext }}$ is the voltage measured at the resistor terminals. During the $t_{\text {off }}$ part of each cycle $R_{\text {ext }}$ is disconnected (zero current) and $U_{\mathrm{MFC}}$ increases. During the $t_{\mathrm{on}}$ part of the cycle $\mathrm{R}_{\text {ext }}$ is re-connected and $U_{\mathrm{MFC}}$ exponentially decreases approaching about $0.14 \mathrm{~V}$ by the end of the cycle. An average (per cycle) power is calculated with respect to both parts of the cycle according to Eq. 2 . In the test presented in Fig. 3, a $P_{\text {av }}$ value of $1.73 \pm 0.03 \mathrm{~mW}$ was obtained. This value was less than the steady state power output of $2.1 \mathrm{~mW}$ obtained with a constantly connected $20 \Omega$ resistance, because of non-optimal cycle parameters. Nevertheless, the test demonstrated that by periodically connecting and disconnecting $R_{\text {ext }}$ a MFC could be operated without significant losses in power output even at $R_{\text {ext }}$ values below $R_{\text {int }}$.

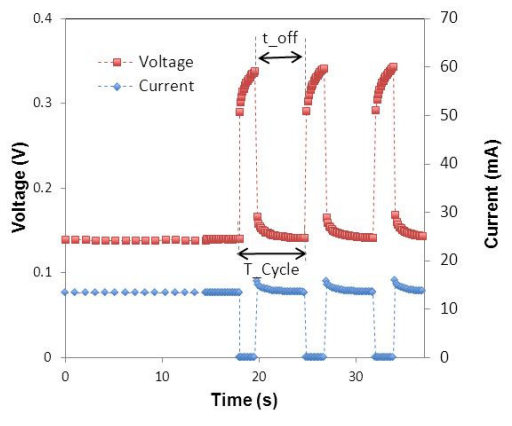

Figure 3. MFC operation with intermittent resistance connection $\left(R_{\mathrm{ext}}=10 \Omega\right)$.

\subsection{D-curves and factorial design tests}


Power output obtained during MFC operation with periodic connection of $\mathrm{R}_{\mathrm{ext}}$ might depend on the choice of cycle parameters $\left(t_{\text {on }}\right.$ and $\left.t_{\text {off }}\right)$, which define the duty cycle value. To study the impact of $D$ on MFC power output the MFC was operated at several $D$ values using a $10 \Omega$ external resistance. For this test a constant value of $t_{o n}=3 \mathrm{~s}$ was used, while $t_{\text {off }}$ was varied to obtain different $D$ values. Fig. 4 shows the impact of $D$ on the average (per cycle) power output. Data points in this curve were obtained by MFC operation at each $D$ value for 8-12 hours. A clear optimum was observed at $D$ values between $0.75-0.95 . P_{\text {av }}$ was maximized at $\mathrm{t}_{\mathrm{on}}=3 \mathrm{~s}$ and $\mathrm{t}_{\text {off }}=0.6 \mathrm{~s}$. Notably, a $P_{\mathrm{av}}$ value of $2 \mathrm{~mW}$ obtained at these cycle settings was quite close to $2.1 \mathrm{~mW}$ measured with a constantly connected $20 \Omega$ resistor. We hypothesize that $P_{a v}$ could be improved by using shorter cycles (i.e. increasing the frequency), but because of hardware limitations we were unable to carry out this test. At $t_{\text {off }}$ values below $0.2 \mathrm{~s}$ the number of voltage samples was insufficient for an accurate estimation of $P_{\mathrm{av}}$.

Additional optimization of cycle parameters ( $t_{\text {on }}$ and $\left.t_{\text {off }}\right)$ was performed using the factorial design experiment. For practical purposes it was decided to switch between the "on" and "off" periods of each cycle by using voltage measurements rather than the pre-selected length of $t_{\mathrm{on}}$ and $t_{\text {off }}$. Indeed, voltage profiles in Fig. 3 always decrease during the $t_{\mathrm{on}}$ part of the cycle until reaching a steady state value. Thus, "on" (resistor is connected) part of the cycle can end if voltage decreases below a pre-set minimum threshold value $\left(U_{\min }\right)$. When $R_{\text {ext }}$ is disconnected the voltage increases until approaching the OCV value. A pre-set maximum voltage threshold $\left(U_{\max }\right)$ can be used to end the "off" (disconnected $R_{\text {ext }}$ ) part of the cycle. The optimal $U_{\min }$ and $U_{\max }$ values were estimated in the full factorial design experiments.

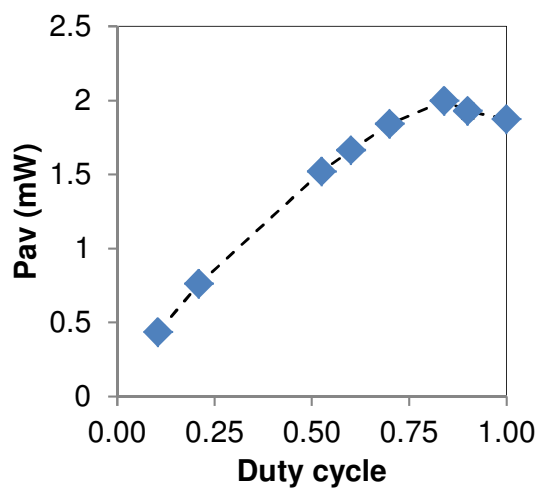

Figure 4. Average power output as a function of the duty cycle at $\mathrm{R}_{\mathrm{ext}}=10.5 \Omega$.

The factorial design experiment was carried out at an $R_{\text {ext }}=10$ $\Omega$ and an OLR of $4 \mathrm{~g} \mathrm{~L}_{\mathrm{A}}^{-1} \mathrm{~d}^{-1}$.. The response surface (Fig. 5) was approximated using a second-order regression model. Statistical analysis of the regression coefficients showed that only linear interactions of the factors should be included. The resulting model had an $R_{\text {adj }}^{2}$ of 0.87 and a $p$-value of 0.94 , i.e. the model was adequate. The optimal operating point was found at a $U_{\max }=0.32 \mathrm{~V}$ and $U_{\min }=0.134 \mathrm{~V}$. This corresponds to a low $U_{\max }$ and a high $U_{\min }$ boundary, i.e. shorter cycles are preferable. This conclusion is consistent with our initial analysis suggesting that shorter cycles lead to optimal power production.

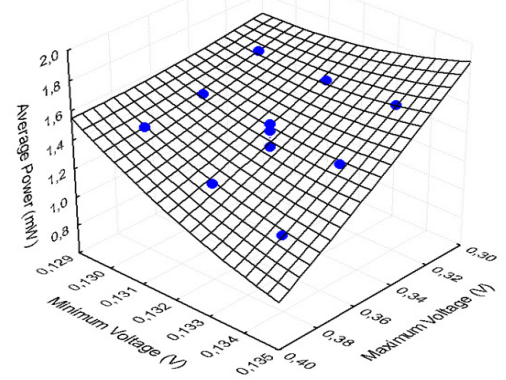

Figure 5. Response surface obtained in the 3-level factorial design test performed at $R_{\mathrm{ext}}=10 \Omega$.

\section{4 -periodic operation under varying organic load}

Estimation of $R_{\text {int }}$ at "normal" and "low" OLRs demonstrated a significant increase of $R_{\text {int }}$ under carbon source - limiting conditions. This change of $R_{\text {int }}$ requires electrical load to be adjusted in order to avoid MFC operation at $R_{\text {ext }}$ values significantly below the $R_{\text {int }}$ value and, consequently, a loss of power output (Pinto et al. 2011; Woodward et al. 2010). MFC operation with periodic connection of $R_{\text {ext }}$ enables MFC operation at $R_{\text {ext }}$ values below $R_{\text {int }}$. Furthermore, duty cycle control based on voltage measurements, as described above, provides a real-time algorithm for maximizing MFC power output.

The robustness of the proposed approach was tested in a MFC, which was first operated with an acetate load of $4 \mathrm{~g} \mathrm{~L}_{\mathrm{A}}^{-1} \mathrm{~d}^{-1}$ followed by a transition to $1 \mathrm{~g} \mathrm{~L}_{\mathrm{A}}^{-1} \mathrm{~d}^{-1}$ to create carbon source limiting conditions. This test was repeated several times, each time using a different method for controlling $R_{\text {ext }}$. Throughout the first test, $R_{\text {ext }}$ was kept at $20 \Omega$. This choice of $R_{\text {ext }}$ maximized power output at acetate non-limiting conditions, however a drop in $P_{\text {av }}$ was observed as soon as acetate concentration in the anodic liquid declined (Fig. 6A). Furthermore, an attempt to change $R_{\text {ext }}$ to $10 \Omega$ during the acetate-limiting phase resulted in an even lower power output (Fig. 6A, a $10 \Omega$ operation is indicated by the arrow).
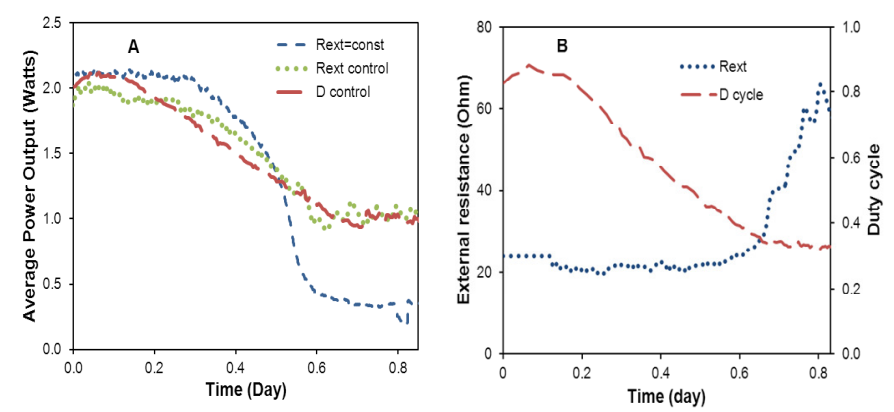

Figure 6. Average power output (A) and $R_{\text {ext }}$ or duty cycle values (B) during organic load perturbation. MFC was 
operated either with a constant $\mathrm{R}_{\mathrm{ext}}=20 \Omega(10 \Omega$ test is indicated by an arrow), or with the $\mathrm{P} / \mathrm{O}$ algorithm for $\mathrm{R}_{\mathrm{ext}}$ control, or with intermittent connection of $\mathrm{R}_{\mathrm{ext}}=10 \Omega$.

During the second test the same acetate load profile was used, while the external resistance was controlled by the $\mathrm{P} / \mathrm{O}$ algorithm described in Woodward et al (Woodward et al. 2010). Real-time optimization of $R_{\text {ext }}$ led to a higher power output at low OLR (Fig. 6A). $R_{\text {ext }}$ chosen by the P/O algorithm was at around $20 \Omega$ under acetate non-limiting conditions, while it increased to 50-70 $\Omega$ during the acetate-limiting phase of the test (Fig. 6B). Notably, the P/O algorithm required $R_{\text {ext }}$ to be adjusted every minute to track the changes in $R_{\text {int }}$.

In the third test the MFC was operated with an intermittently connected $R_{\text {ext }}$ of $10 \Omega$. Voltage threshold values were set to $0.12 \mathrm{~V}$ and $0.32 \mathrm{~V}$ for $U_{\min }$ and $U_{\max }$, respectively. The resulting profile of $P_{\mathrm{av}}$ is shown in Fig. 6A. It can be seen, that by intermittently connecting the electrical load based on the voltage threshold, the power output was maximized both under acetate non-limiting and non-limiting conditions without changing the value of the external resistance. The corresponding duty cycle values are shown in Fig. 6B. It can be seen that at a low acetate concentrations, the $D$ values increased, i.e. $R_{\text {ext }}$ was connected for a shorter time during each cycle. Considering that for practical MFC application for electricity production MFC output voltage should be converted to at least $5 \mathrm{~V}$, e.g. using an up-converter, this approach would also allow for better up-converter performance. Although upconverters can operate at low input voltages, the conversion efficiency decreases if the input voltage drops below $100 \mathrm{mV}$. Consequently, D-cycle adaptation based on voltage thresholds might improve the overall system efficiency.

\section{CONCLUSIONS}

This study demonstrates that an MFC can be operated at electrical loads below the MFC internal resistance without significant energy losses and proposes a practical method for maximizing MFC power output. The problem of $R_{\text {ext }}$ and $R_{\text {int }}$ mismatch is resolved by MFC operation with periodic resistance connection, where the connection and disconnection times are controlled based on the voltage measurements. The proposed approach not only resolves the problem of resistance mismatch but also accounts for the changes in MFC electrochemical characteristics due to variations in operating conditions.

\section{REFERENCES}

Aelterman, P., M. Versichele, M. Marzorati, N. Boon and W. Verstraete. 2008. "Loading rate and external resistance control the electricity generation of microbial fuel cells with different three-dimensional anodes." Biores. Technol. 99: 8895-8902.

Chen, S., G. He, A. A. Carmona-Martinez, S. Agarwal, A. Greiner, H. Hou and U. Schröder. 2011. "Electrospun carbon fiber mat with layered architecture for anode in microbial fuel cells." Electrochem. Communications 13:1026-1029.

Donovan, C., A. Dewan, D. Heo and H. Beyenal. 2008. "Batteryless, Wireless Sensor Powered by a Sediment
Microbial Fuel Cell." Environmental Science and Technolofy 42:8591-8596.

Esram, T. and P.L. Chapman. 2007. "Comparison of Photovoltaic Array Maximum Power Point Tracking Techniques " IEEE TRANSACTIONS ON ENERGY CONVERSION 22:439-449.

Fan, Y., E. Sharbrough and H. Liu. 2008. "Quantification of the internal resistance distribution of microbial fuel cells." Environmental Science and Technology 42:8101-8107.

Koutroulis , E. and K. Kalaitzakis 2006. "Design of a Maximum Power Tracking System for Wind-EnergyConversion Applications " IEEE TRANSACTIONS ON INDUSTRIAL ELECTRONICS 53:486-494.

Logan, B. E. 2008. Microbial Fuel Cells. Hoboken, NJ: John Wiley \& Sons Inc.

Logan, B.E. and J.M. Regan. 2006. "Electricity-producing bacterial communities in microbial fuel cells." Trends in Microbiology 14:512-518.

Lovley, D.R. 2006. "Bug juice: harvesting electricity with microorganisms." Nature Reviews Microbiology 4(7):497508.

Pinto, R. P., B. Srinivasan, S.R. Guiot and B. Tartakovsky. 2011. "The Effect of Real-Time External Resistance Optimization on Microbial Fuel Cell Performance." Water Research 45:1571-1578.

Premier, G.C., J.R. Kim, I. Michie, R.M. Dinsdale and A.J. Guwy. 2011. "Automatic control of load increases power and efficiency in a microbial fuel cell." J. Power Sources 196 2013-2019.

Rismani-Yazdi, H., S.M. Carver, A.D. Christy and I.H. Tuovinen. 2008. "Cathodic limitations in microbial fuel cells: An overview." J. Power Sources 180:683-694.

Rozendal, R.A., H.V.M. Hamelers, K. Rabaey, J. Keller and C.J.N. Buisman. 2008. "Towards practical implementation of bioelectrochemical wastewater treatment." Trends in Biotechnol. 26:450-459.

Trasatti, S. and O.A. Petrii. 1991. "Real surface area measurements in electrochemistry." Pure \& Appl. Chern. 63:711-734.

Woodward, L., M. Perrier, B. Srinivasan, R.P. Pinto and B. Tartakovsky. 2010. "Comparison of real-time methods for maximizing power output in microbial fuel cells." AIChE J. 56:2742-2750.

Woodward, L., B. Tartakovsky, M. Perrier and B. Srinivasan. 2009. "Maximizing power production in a stack of microbial fuel cells using multiunit optimization method." Biotechnol. Prog. 25(3):676-682. 